\title{
Prevalence of mental disorders among people who are homeless: an umbrella review
}

\author{
Md Mahbub Hossain, ${ }^{\mathrm{a}}$ Abida Sultana, ${ }^{\mathrm{b}}$ Samia Tasnim, ${ }^{\mathrm{a}}$ Qiping Fan, ${ }^{\mathrm{a}}$ Ping Ma, ${ }^{\mathrm{a}}$ \\ E. Lisako J. McKyer, ${ }^{a}$ Neetu Purohit ${ }^{\mathrm{c}}$
}

\begin{abstract}
Introduction: Homelessness is a major problem that critically impacts the mental health and wellbeing of the affected individuals. This umbrella review aimed to evaluate the current evidence on the prevalence of mental disorders among homeless people from evidence-based systematic reviews and meta-analyses.

Methods: We followed the Preferred Reporting Items for Systematic Reviews and MetaAnalyses (PRISMA) guidelines and Joanna Briggs Institute (JBI) methodology for umbrella reviews. We searched 12 major databases and additional sources to identify systematically conducted reviews and meta-analyses reporting the prevalence of mental disorders among homeless populations.
\end{abstract}

Results: We evaluated 1,277 citations and found 15 reviews meeting our criteria. Most studies were conducted among high-income countries with samples from different age groups. Studies reported high prevalence rates of depressive and anxiety disorders, schizophrenia spectrum and psychotic disorders, substance use disorders, suicidal behavior, bipolar and mood disorders, neurocognitive disorders, and other mental disorders among homeless people. Moreover, studies also reported a high burden of co-occurring mental and physical health problems among the homeless experiencing mental disorders.

Conclusions: This umbrella review synthesized the current evidence on the epidemiological burden of mental disorders in homelessness. This evidence necessitates advanced research to explore psychosocial and epidemiological correlates and adopt multipronged interventions to prevent, identify, and treat mental disorders among homeless populations.

Keywords: Mental Disorders; Psychiatric Disorders; Mental Health; Mental Health Services; Homeless; Homelessness; Systematic Review; Meta-analysis; Umbrella Review; Evidence-based practice

\footnotetext{
a. Department of Health Promotion and Community Health Sciences, School of Public Health, Texas A\&M University, TX 77843, USA.

b. Nature Study Society of Bangladesh, Khulna 09000, Bangladesh.

c. The IIHMR University, Jaipur, Rajasthan 302029, India.

*Corresponding author: Md Mahbub Hossain, School of Public Health, Texas A\&M University, TX 77843, USA.

Email: mhossain@tamu.edu, mhossa13@alumni.jh.edu
} 


\section{Introduction}

Homelessness is a growing population health concern worldwide (Omerov, Craftman, Mattsson, \& Klarare, 2020). In the United States, a total of 552,830 people experienced homelessness on a single night in 2018 (Henry et al., 2018). In the same year, nearly 320,000 people in the United Kingdom were recorded as homeless (Shelter England, 2018). Moreover, around 235,000 individuals were homeless during 2016 in Canada (Gaetz, Dej, Richter, \& Redman, 2016). In the twentieth century, homeless people were typically older men, whereas homelessness in recent years has been observed among females and youth of different racial and ethnic groups (Jones, 2016). This evidence provides an overall scenario of homelessness in the high-income nations; however, evidence on the severity of homelessness is scarce from low- and middle- income countries (LMICs). Socioeconomic factors associated with homelessness including rapid urbanization and industrialization, high poverty, income inequality, unemployment, maldistribution of resources between rural and urban areas, migration, and lack of access to affordable housing are prevalence in LMICs, which suggest the magnitude of homelessness is likely to be higher in those contexts (Speak, 2019). This global burden of homelessness has critical implications for health policymaking and practice. In homelessness, the rate of mortality is nearly eight times higher than the average for men and 12 times higher for women, with an average age for death at 52 years (Aldridge et al., 2019, 2018). This can be attributable to the fact that homeless individuals experience enormous health inequalities and have a higher prevalence of various medical conditions (Omerov et al., 2020). This burden increases among aging people who are homeless. They often experience multiple health problems, which become worse in the presence of a lack of access to healthcare services, poor social ties, and continued effects of other social determinants of health (Omerov et al., 2020). In most cases, such problems are not prevented or diagnoses at earlier stages resulting in increased use of acute care services and higher cost to the health systems (Omerov et al., 2020; Rosenheck \& Seibyl, 1998).

Addressing homelessness is essential for the overall health and wellbeing of a population. This agenda is part of the United Nations (UN) 2030 Agenda for Sustainable Development (United Nations, 2015). The action plans to alleviate homelessness include reducing poverty and ensuring health and wellbeing. In addition to addressing residential challenges and physical health problems, it is necessary to improve the mental health of people who are homeless 
(Altena, Brilleslijper-Kater, \& Wolf, 2010; Dickey, 2000). Psychosocial stressors like impaired interpersonal relationships, lack of hope, loneliness, and poor social capital affect the mental health and wellbeing during homelessness (Omerov et al., 2020). Another challenge is the deinstitutionalization of mental illness in many countries like the United States, which resulted in a reduced number of beds in indoor psychiatric facilities without strengthening community shelters and models of care (Yohanna, 2013). Additionally, LMICs generally have lesser organizational capacities to provide mental health services, both in the institutional and community settings (Daund, Sonavane, Shrivastava, Desousa, \& Kumawat, 2018). Such challenges may have brought many people with severe mental illness to the temporary shelters and streets, thus increasing both the burden of mental disorders and homelessness within a given geographic region. From a population health perspective, homeless people, irrespective of a previous psychiatric diagnosis, are likely to live in disadvantaged conditions that make them vulnerable to mental disorders and remain undiagnosed as well as untreated in most cases (Patten, 2017; Yim, Leung, Chan, Lam, \& Lim, 2015).

To address the pre-existing mental disorders and promote positive mental health and resilience, it is essential to understand the epidemiological burden of mental disorders among homeless individuals. In this regard, observational studies may provide insights about the spatial and temporal distribution of mental disorders in homelessness. Moreover, systematic reviews or meta-analysis of observational studies can address the sampling errors of individual studies and provide a less-biased estimate of the burden of health conditions of interest. Given that more than 11 systematic reviews or meta-analyses are published in a day (Bastian, Glasziou, \& Chalmers, 2010), it is essential to synthesize the evidence from existing reviews. Such reviews of the reviews, also known as umbrella reviews, have shown advantages over meta-analyses due to their bird's eye view of evidence (Ioannidis, 2009). Furthermore, umbrella reviews are increasingly used for clinical risk prediction and evidence-based actions through synthesizing prior knowledge on several mental disorders (Fullana et al., 2019). There is a lack of an umbrella review that can provide global evidence on the prevalence of mental disorders in homelessness, which may inform future research, policymaking, and practice. This umbrella review acknowledged this knowledge gap and synthesized the current evidence on the prevalence of mental disorders among people who are homeless. 


\section{Materials and methods}

\section{Search strategy of the review}

This umbrella review was conducted in accordance with the Preferred Reporting Items for Systematic Reviews and Meta-Analyses (PRISMA) guidelines (Liberati et al., 2009), and the Joanna Briggs Institute (JBI) methodology for umbrella reviews (Aromataris et al., 2015). We searched MEDLINE, Embase, PubMed, PsycINFO, CINAHL, Health Policy Reference Center, ERIC, Health Source (Nursing/Academic Edition), Environment Complete, Child Development \& Adolescent Studies, Academic Search Ultimate, and the Cochrane Library using specific keywords (please see Table 1). For each database, the titles, abstracts, subject headings, and general keywords were searched with no language or time restrictions. Moreover, we searched the citations used as references of the primarily screened articles and citing articles from Google Scholar using the "cited by" function. All databases and additional sources were searched from their inception to October 15, 2019, and the entire search process was repeated on December 21, 2019, for the last time. All the citations were compiled using RefWorks software and uploaded to Rayyan cloud-based citations management system for systematic evaluation.

\section{Inclusion and exclusion criteria}

In this umbrella review, we included reviews that systematically evaluated and reported the prevalence of mental disorders among people who are homeless. In addition, studies reporting other quantitative measures of disease burden (for example, odds ratio or relative risk expressing the epidemiological burden of a disorder) were also considered in the absence of a prevalence value. To specify mental disorders, we adopted the classifications of mental disorders in the International Classification of Diseases (ICD) tenth revision (World Health Organization (WHO), 2016), or the Diagnostic and Statistical Manual (DSM) fifth edition (American Psychiatric Association, 2013). In addition, we acknowledged the historical evolution of the 
Table 1: Search strategy for this umbrella review

\begin{tabular}{|c|c|}
\hline Search query & $\begin{array}{l}\text { Keywords (searched within titles, abstracts, subject headings like MeSH, } \\
\text { and general keywords) }\end{array}$ \\
\hline 1 & $\begin{array}{l}\text { prevalence OR incidence OR epidemiology OR frequency OR cases OR odds } \\
\text { OR risks OR status OR "risk factors" OR "protective factors" OR "associated } \\
\text { factors" OR correlates OR predictors OR distribution OR determinants }\end{array}$ \\
\hline 2 & 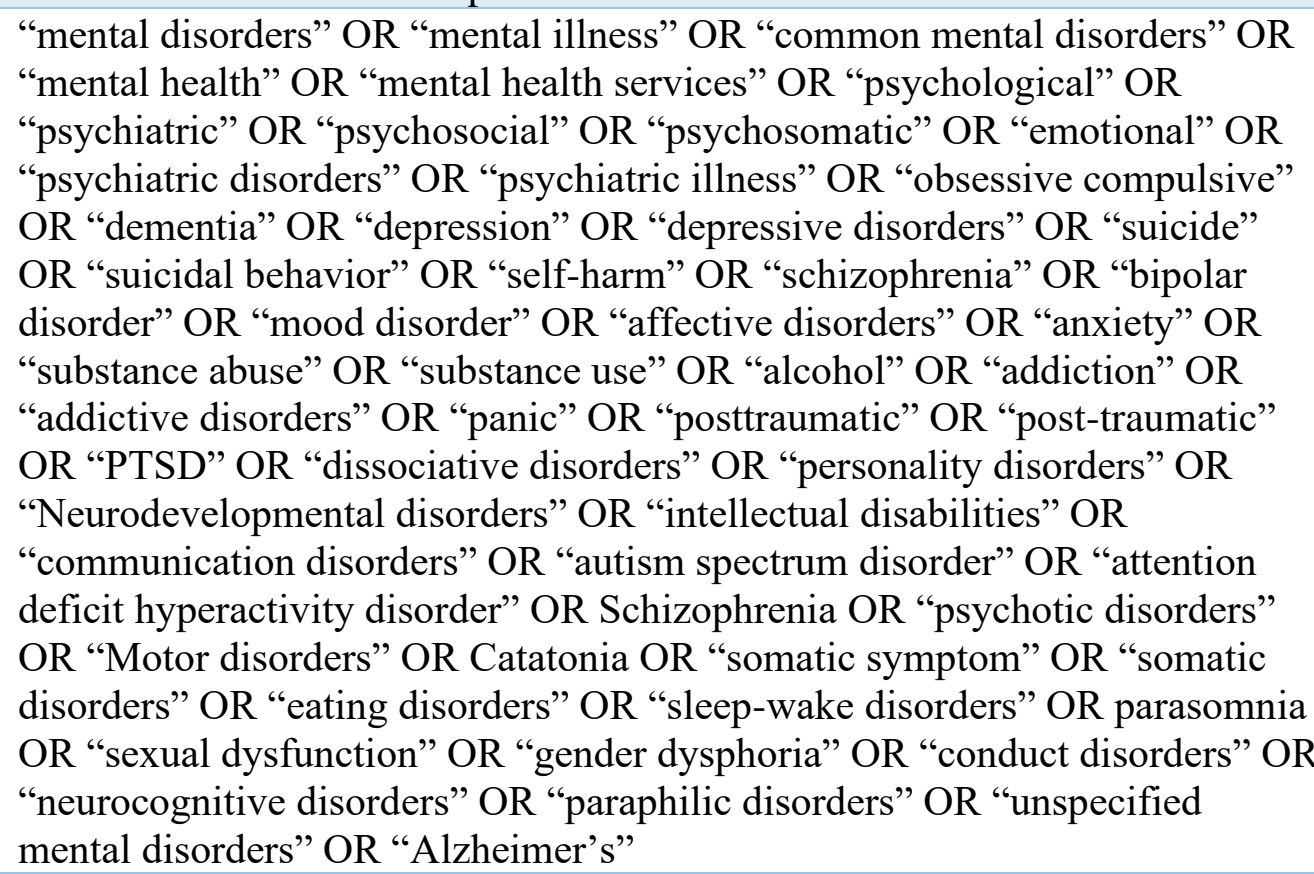 \\
\hline 3 & $\begin{array}{l}\text { systematic review OR meta-analysis OR meta-regression OR pooled effect } \\
\text { OR pooled estimate }\end{array}$ \\
\hline 4 & Homeless OR homelessness OR unsheltered \\
\hline $\begin{array}{l}\text { Complete } \\
\text { query }\end{array}$ & 1 AND 2 AND 3 AND 4 \\
\hline
\end{tabular}

definitions and conceptual constructs within mental disorders. Therefore, we included reviews reporting mental disorders which are consistent with the earlier versions of ICD or DSM and have an equivalent diagnosis classified under the current versions of these guidelines. Moreover, there are several definitions of homelessness in different contexts, which may include some individuals while excluding others within the scope of respective definitions (Byrne \& Culhane, 2015; US Health and Human Services, 2020). For example, in the United States, two major definitions are used by federal agencies to identify the homeless population and provide social care. While both definitions agree on most criteria, individuals living in motels or staying with others lacking a regular accommodation are considered homeless by the educational program definition, whereas those individuals are not eligible as homeless as per the Housing and Urban 
Development (HUD) definition (US Health and Human Services, 2020). Such definitions may have provided different estimations of study samples and the magnitude of different health problems in those samples. This review acknowledged such differences and considered any definition of homelessness in the eligible reviews and respective primary studies, which allowed this review to remain inclusive to diverse operational definitions that may have been used in different contexts over time.

Furthermore, articles were recruited if they fulfilled all the inclusion criteria and excluded if they did not meet any of the exclusion criteria as listed in Table 2. In this umbrella review, two authors independently evaluated the citations according to the pre-specified criteria. Any conflicts arising in the screening process were resolved at the end of the independent screening through discussion in the presence of a third author.

\section{Table 2: Eligibility criteria for this review}

\begin{tabular}{|l|l|}
\hline \multicolumn{1}{|c|}{ Inclusion criteria } \\
$\begin{array}{l}\text { 1. Systematically conducted narrative, } \\
\text { qualitative, scoping, or quantitative } \\
\text { (meta-analytic) literature reviews }\end{array}$ & $\begin{array}{l}\text { 1. Articles which were not systematically } \\
\text { conducted reviews (for example, unstructured } \\
\text { reviews without a plausible methodology, primary } \\
\text { studies, opinions, commentaries, letters, or } \\
\text { editorials were excluded from this review) }\end{array}$ \\
\hline $\begin{array}{l}\text { 2. Articles reported prevalence (or other } \\
\text { quantitative measures like incidence, } \\
\text { cases with denominators, odds, or } \\
\text { relative risks etc.) of mental disorders as } \\
\text { specified within current or earlier } \\
\text { versions of ICD or DSM }\end{array}$ & $\begin{array}{l}\text { 2. Articles which did not specifically report the } \\
\text { prevalence (or other quantitative measures) of } \\
\text { mental only were excluded (for example, articles } \\
\text { reporting mental disorders within general health } \\
\text { outcomes were excluded) }\end{array}$ \\
\hline $\begin{array}{l}\text { 3. Articles that primarily focused on the } \\
\text { homeless people, irrespective of } \\
\text { definitions of homelessness in those } \\
\text { studies }\end{array}$ & $\begin{array}{l}\text { 3. Articles reported primary studies conducted in } \\
\text { general population or mixed population groups } \\
\text { without a focus on homeless people (for example, } \\
\text { mental disorders among participants including } \\
\text { some homeless individuals were excluded, unless } \\
\text { they reported the prevalence among homeless } \\
\text { individuals as an identifiable group) }\end{array}$ \\
\hline $\begin{array}{l}\text { 4. Published as peer-reviewed journal } \\
\text { articles }\end{array}$ & $\begin{array}{l}\text { 4. Articles, dissertations, theses, policy papers, or } \\
\text { institutional reports were excluded if they were } \\
\text { not published as peer-reviewed articles in journals }\end{array}$ \\
\hline $\begin{array}{l}\text { 5. Full texts were available in the English } \\
\text { language }\end{array}$ & $\begin{array}{l}\text { 5. Articles were excluded if the full texts were not } \\
\text { available in the English language }\end{array}$ \\
\hline
\end{tabular}




\section{Data extraction and synthesis}

A data extraction tool was developed adopting the JBI data extraction tool for systematic reviews and research synthesis (Munn, Tufanaru, \& Aromataris, 2014). Two authors used this tool and independently extracted data on the following domains: the objectives and types of each review, year of publication, names of databases searched in respective reviews, the timeframe of searching databases, sample size, location of the primary studies, demographic characteristics of the participants, recruitment strategy, and key findings on the prevalence of mental disorders among homeless people. Furthermore, a narrative synthesis of the research findings was conducted considering high heterogeneity in terms of operational definitions of homelessness as well as mental disorders, methodological approaches, and instruments within the primary studies and reviews. The synthesized findings on the prevalence rates (percentage, proportion, odds ratio [OR], relative risk [RR], or other quantitative measures) with specific or range estimations within $95 \%$ confidence interval $[\mathrm{CI}]$ were reported from the respective reviews.

\section{Evaluation of the methodological quality}

To evaluate the methodological quality of the reviews, we used the JBI critical appraisal checklist for systematic reviews and research synthesis checklist (Aromataris et al., 2015). Two reviewers independently evaluated each of the included reviews. At the end of the primary evaluation, two reviewers discussed the evaluation findings, reached a consensus for all the items, and finalized the overall quality ratings. The checklist consists of ten items, and each item could receive one point. Therefore, the overall quality score of a review could range from zero to ten. In this umbrella review, articles receiving zero to four, five to seven, and eight to ten were categorized as the low, medium, and high-quality studies, respectively. 


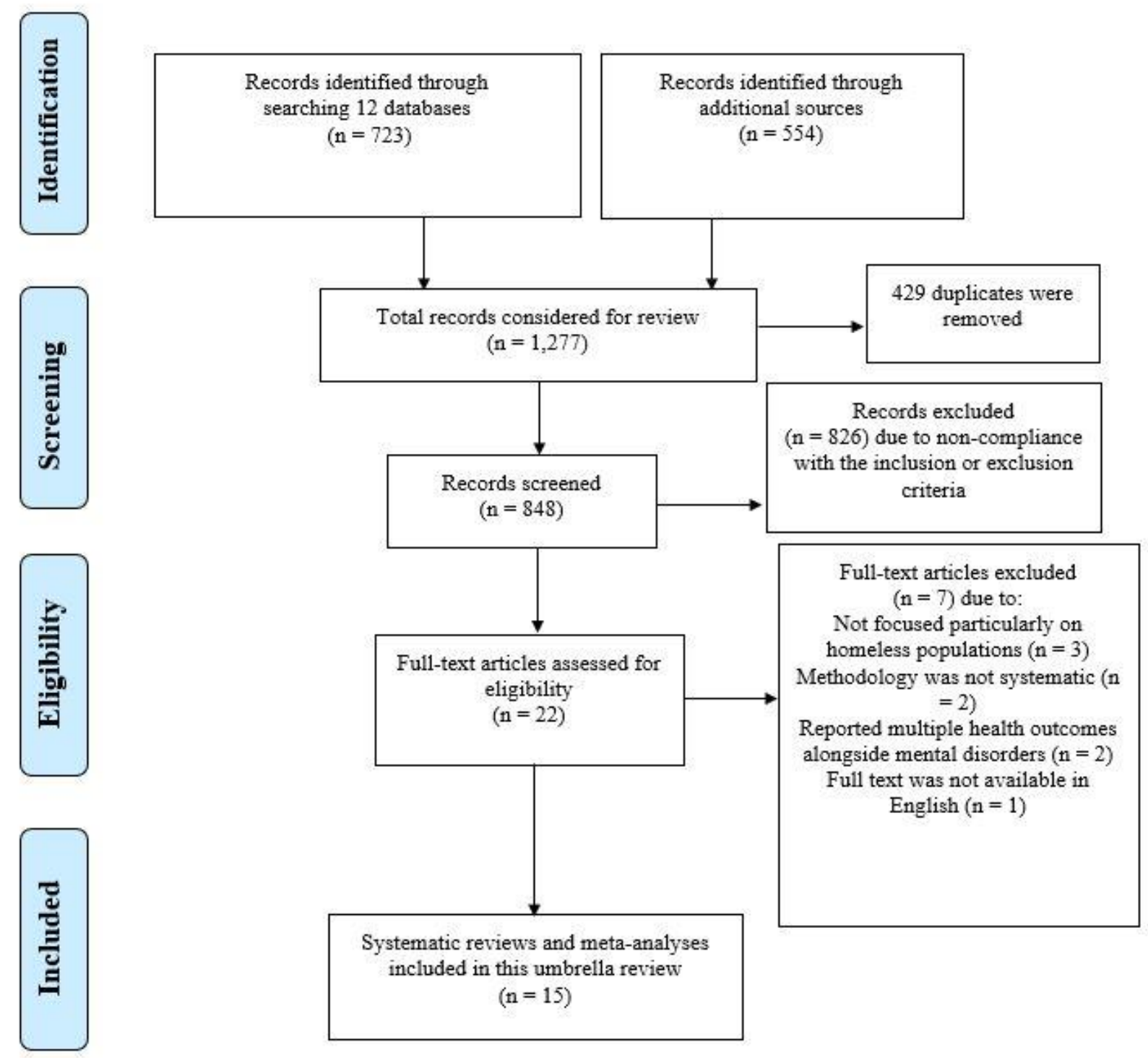

Figure 1: Flow diagram of the umbrella review

\section{Results}

We found 723 citations from searching 12 databases and 554 citations from additional sources, totaling 1,277 citations (please see Figure 1). After eliminating 429 duplicates, we evaluated the titles and abstracts of the remaining 848 citations as per the pre-specified criteria for this review. At the end of this stage, we removed 826 citations due to non-compliance with our criteria and evaluated full texts of the remaining 22 citations. Seven articles were excluded at this stage, and we retained 15 articles in this review (Ayano, Tesfaw, \& Shumet, 2019; Ayano, Tsegay, Abraha, \& Yohannes, 2019; Bassuk, Richard, \& Tsertsvadze, 2015; Burra, Stergiopoulos, \& Rourke, 
2009; Depp, Vella, Orff, \& Twamley, 2015; Duke \& Searby, 2019; Embleton, Mwangi, Vreeman, Ayuku, \& Braitstein, 2013; Ennis, Roy, \& Topolovec-Vranic, 2015; Fazel, Khosla, Doll, \& Geddes, 2008; Folsom \& Jeste, 2002; Hodgson, Shelton, van den Bree, \& Los, 2013; Parks, Stevens, \& Spence, 2007; Schreiter et al., 2017; Smartt et al., 2019; Spence, Stevens, \& Parks, 2004). The included articles consisted of seven meta-analytic reviews (Ayano, Tesfaw, et al., 2019; Ayano, Tsegay, et al., 2019; Bassuk et al., 2015; Depp et al., 2015; Embleton et al., 2013; Fazel et al., 2008; Schreiter et al., 2017), and eight non-quantitative reviews (Burra et al., 2009; Duke \& Searby, 2019; Ennis et al., 2015; Folsom \& Jeste, 2002; Hodgson et al., 2013; Parks et al., 2007; Smartt et al., 2019; Spence et al., 2004). The summary findings of the included reviews are provided in Table 3.

\section{Characteristics of the included reviews}

Most reviews $(n=10)$ included primary studies from multiple countries without specific geographic or economic focus, whereas three reviews emphasized on high-income countries (Bassuk et al., 2015; Fazel et al., 2008; Schreiter et al., 2017) and two reviews included studies from LMICs or resource-constrained contexts (Embleton et al., 2013; Smartt et al., 2019). Among reviews without a contextual focus, most primary studies were in the US, Germany, Canada, UK, Australia, and other developed countries with fewer studies from LMICs (Ayano, Tesfaw, et al., 2019; Ayano, Tsegay, et al., 2019; Burra et al., 2009; Depp et al., 2015; Folsom \& Jeste, 2002). Moreover, the earliest review was published in 2002, and a total of five reviews were published before 2010 (Burra et al., 2009; Fazel et al., 2008; Folsom \& Jeste, 2002; Parks et al., 2007; Spence et al., 2004), whereas most $(n=10)$ reviews were published after 2010. Furthermore, the median number of databases searched in the respective reviews was three, with a range from two to 16 . The number of primary studies included in the respective reviews ranged from 10 to 50. In addition, the evaluation of the methodological quality (please see Supplementary file) found seven reviews with medium quality (Embleton et al., 2013; Ennis et al., 2015; Folsom \& Jeste, 2002; Hodgson et al., 2013; Parks et al., 2007; Schreiter et al., 2017; Spence et al., 2004) and eight reviews with high methodological quality (Ayano, Tesfaw, et al., 2019; Ayano, Tsegay, et al., 2019; Bassuk et al., 2015; Burra et al., 2009; Depp et al., 2015; Duke \& Searby, 2019; Fazel et al., 2008; Smartt et al., 2019). 
Table 3: Characteristics and the key findings of the included systematic reviews and meta-analyses

\begin{tabular}{|c|c|c|c|c|c|}
\hline Source & $\begin{array}{l}\text { Name(s) and } \\
\text { timeframe of } \\
\text { searching } \\
\text { Databases }\end{array}$ & $\begin{array}{l}\text { Number and } \\
\text { type of } \\
\text { primary } \\
\text { studies; } \\
\text { meta- } \\
\text { analysis or } \\
\text { systematic } \\
\text { review }\end{array}$ & $\begin{array}{l}\text { Country of the } \\
\text { study } \\
\text { population }\end{array}$ & $\begin{array}{l}\text { Sample size and } \\
\text { characteristics of } \\
\text { the study } \\
\text { population (if } \\
\text { reported) }\end{array}$ & $\begin{array}{l}\text { Prevalence rates (or other quantitative } \\
\text { measures of the epidemiological burden as } \\
\text { specified) and related key findings }\end{array}$ \\
\hline $\begin{array}{l}\text { (Folsom \& } \\
\text { Jeste, } \\
\text { 2002) }\end{array}$ & $\begin{array}{l}\text { MEDLINE, } \\
\text { PsycINFO, and } \\
\text { additional sources; } \\
\text { Post-1966 }\end{array}$ & $\begin{array}{l}33 ; \\
\text { Systematic } \\
\text { review }\end{array}$ & $\begin{array}{l}\text { Most }(\mathrm{n}=16) \\
\text { from the US, } \\
\text { remaining } \\
\text { studies were } \\
\text { from the US ( } \mathrm{n} \\
=7) \text {, Germany } \\
(\mathrm{n}=3), \\
\text { Australia }(\mathrm{n}= \\
\text { 2), Canada ( } \mathrm{n}= \\
\text { 2), and one } \\
\text { study each from } \\
\text { Brazil, Spain, } \\
\text { and France }\end{array}$ & $\begin{array}{l}\text { Sample size ranged } \\
\text { from } 32 \text { to } 27,638 ; \\
\text { most samples had a } \\
\text { higher proportion of } \\
\text { male and adult } \\
\text { participants; samples } \\
\text { were recruited from } \\
\text { diverse settings } \\
\text { including } \\
\text { downtown, suburban } \\
\text { areas, rural counties, } \\
\text { jails, hostels, and } \\
\text { homeless shelters }\end{array}$ & $\begin{array}{l}\text { The overall prevalence of schizophrenia ranged } \\
\text { from } 1 \% \text { to } 45 \% \text {. Studies with higher quality } \\
\text { had a weighted prevalence of } 11 \% \text { (range: } 4 \%- \\
16 \% \text { ). Young participants had a higher } \\
\text { prevalence (ranged } 13 \%-21 \% \text { ) compared to } \\
\text { older participants (range: } 8 \%-14 \% \text { ). Women } \\
\text { had a higher overall prevalence than men in } \\
\text { five studies. Newly homeless participants had a } \\
\text { lower prevalence of schizophrenia (range: } 2 \%- \\
14 \% \text { ) compared to chronically homeless } \\
\text { participants (range: } 18 \%-27 \% \text { ) }\end{array}$ \\
\hline $\begin{array}{l}\text { (Spence et } \\
\text { al., 2004) }\end{array}$ & $\begin{array}{l}\text { PsycINFO, } \\
\text { MEDLINE, Embase, } \\
\text { Social Science } \\
\text { Citation Index, } \\
\text { Science Citation } \\
\text { Index, CINAHL, } \\
\text { Biological Abstracts, } \\
\text { ASSIA, INSPEC, } \\
\text { HMIC/ } \\
\text { DH Data, British } \\
\text { Nursing Index, } \\
\text { SPORT Discus, } \\
\text { AMED, Econlit, and } \\
\text { additional sources; }\end{array}$ & $\begin{array}{l}18 ; \\
\text { Systematic } \\
\text { review }\end{array}$ & $\begin{array}{l}\text { Country } \\
\text { statistics was } \\
\text { provided for } 18 \\
\text { studies; } 11 \text { of } \\
\text { them were } \\
\text { conducted in the } \\
\text { US, seven were } \\
\text { from the rest of } \\
\text { the world }\end{array}$ & $\begin{array}{l}\text { The sample size was } \\
\text { not reported for all } \\
\text { the primary studies; } \\
\text { adult homeless were } \\
\text { the focus of the } \\
\text { review; fewer } \\
\text { studies reported } \\
\text { demographics of the } \\
\text { participants, most } \\
\text { were male, with an } \\
\text { over-representation } \\
\text { of ethnic minorities; } \\
\text { most were recruited } \\
\text { from hostels }\end{array}$ & $\begin{array}{l}\text { Nearly } 30-40 \% \text { of homeless adults had } \\
\text { cognitive impairment compared to } 2-3 \% \text { of } \\
\text { adults living in the community. Moreover, } \\
\text { intelligence quotient (IQ) among homeless } \\
\text { adults was lower than the average range. } \\
\text { Studies have also reported co-occurring } \\
\text { psychiatric morbidity including depression, } \\
\text { alcohol dependence, and schizophrenia among } \\
\text { the homeless population }\end{array}$ \\
\hline
\end{tabular}




\begin{tabular}{|c|c|c|c|c|c|}
\hline & $\begin{array}{l}\text { timeframe was not } \\
\text { specified }\end{array}$ & & & & \\
\hline $\begin{array}{l}\text { (Parks et } \\
\text { al., 2007) }\end{array}$ & $\begin{array}{l}\text { PsycINFO, } \\
\text { MEDLINE, Embase, } \\
\text { Social Science } \\
\text { Citation Index, } \\
\text { Science Citation } \\
\text { Index, CINAHL, } \\
\text { Biological Abstracts, } \\
\text { ASSIA, INSPEC, } \\
\text { HMIC/DH Data, } \\
\text { British Nursing } \\
\text { Index, SPORT } \\
\text { Discus, AMED, } \\
\text { Econlit, Index to } \\
\text { Theses, International } \\
\text { Bibliography of the } \\
\text { Social Sciences, and } \\
\text { additional sources; } \\
\text { Post-1966 }\end{array}$ & $\begin{array}{l}10 ; \\
\text { Systematic } \\
\text { review }\end{array}$ & $\begin{array}{l}\text { Most samples } \\
\text { were from the } \\
\text { US, only one } \\
\text { study was from } \\
\text { South Africa }\end{array}$ & $\begin{array}{l}\text { The total sample } \\
\text { was less than } 2,000 \text {; } \\
\text { this review focused } \\
\text { on children and } \\
\text { adolescents; } \\
\text { participants were } \\
\text { mostly male, were } \\
\text { recruited from } \\
\text { shelters }\end{array}$ & $\begin{array}{l}\text { Impaired cognitive functions were found } \\
\text { among the study participants. One study found } \\
80 \% \text { of the participants had } 50^{\text {th }} \text { percentile or } \\
\text { lower language deprivation and learning } \\
\text { difficulties; sub-group analysis reported that } \\
11 \% \text { of children had mild retardation and } 35 \% \\
\text { had learning difficulties }\end{array}$ \\
\hline $\begin{array}{l}\text { (Fazel et } \\
\text { al., 2008) }\end{array}$ & $\begin{array}{l}\text { Embase, MEDLINE, } \\
\text { PsycINFO, and } \\
\text { additional sources; } \\
\text { 1966-2007 }\end{array}$ & $\begin{array}{l}\text { 29; Meta- } \\
\text { analysis }\end{array}$ & $\begin{array}{l}\text { Most studies } \\
\text { were from the } \\
\text { US }(n=10), \\
\text { followed by the } \\
\text { US }(n=8), \\
\text { Germany ( } n= \\
6 \text { ), Australia (n } \\
=2 \text { ), and one } \\
\text { each from } \\
\text { Netherlands, } \\
\text { Greece, and } \\
\text { France }\end{array}$ & $\begin{array}{l}\text { The total sample } \\
\text { was } 5,684 \text {; most } \\
\text { participants were } \\
\text { male ( } 82 \% \text { in the } \\
\text { mixed samples), the } \\
\text { average age of the } \\
\text { participants was } \\
40.1 \text { years in mixed } \\
\text { samples; participants } \\
\text { were recruited from } \\
\text { multiple settings } \\
\text { including shelters, } \\
\text { hostels, day centers, } \\
\text { missions, and streets }\end{array}$ & $\begin{array}{l}\text { Studies reported common mental disorders, } \\
\text { including alcohol dependence (ranged from } \\
8.1 \% \text { to } 58.5 \% \text { ), and drug dependence (ranged } \\
\text { from } 4.5 \% \text { to } 54.2 \% \text { ). For psychotic illness, the } \\
\text { prevalence ranged from } 2.8 \% \text { to } 42.3 \% \text {. The } \\
\text { pooled prevalence of psychotic disorders was } \\
12.7 \% \text { (95\% CI: } 10.2-15.2 \text { ) in } 28 \text { studies. The } \\
\text { prevalence of major depression was } 11.4 \% \\
\text { (95\% CI: } 8.4-14.4 \text { ) in } 19 \text { studies. The pooled } \\
\text { prevalence of personality disorders and alcohol } \\
\text { dependence was } 23.1 \% \text { (95\% CI: } 15.5-30.8 \text { ) } \\
\text { and } 37.9 \% \text { ( } 95 \% \text { CI: } 27.8-48 \text { ) from random- } \\
\text { effects models using data from } 14 \text { and } 10 \\
\text { studies, respectively. Moreover, the pooled } \\
\text { prevalence of drug dependence was } 24.4 \% \\
\text { (95\% CI: } 13.2-35.6 \text { ) in seven studies. Samples }\end{array}$ \\
\hline
\end{tabular}




\begin{tabular}{|c|c|c|c|c|c|}
\hline & & & & & CI: 10-22) \\
\hline $\begin{array}{l}\text { (Burra et } \\
\text { al., 2009) }\end{array}$ & $\begin{array}{l}\text { MEDLINE, } \\
\text { CINAHL, Embase, } \\
\text { PsycINFO, Applied } \\
\text { Social Sciences } \\
\text { Index and Abstracts, } \\
\text { ERIC, Social } \\
\text { Sciences Abstracts, } \\
\text { Social Sciences } \\
\text { Citation Index, } \\
\text { Social Services } \\
\text { Abstracts, } \\
\text { Sociological } \\
\text { Abstracts, and } \\
\text { additional sources; } \\
\text { 1970-2007 }\end{array}$ & $\begin{array}{l}22 ; \\
\text { Systematic } \\
\text { review }\end{array}$ & $\begin{array}{l}\text { Most studies ( } \mathrm{n} \\
=13 \text { ) were from } \\
\text { the US followed } \\
\text { by the US ( } \mathrm{n}= \\
3) \text {, Australia ( } \mathrm{n} \\
=2) \text {, Brazil ( } \mathrm{n}= \\
2) \text {, Germany (n } \\
=2) \text {, and Spain } \\
(\mathrm{n}=1)\end{array}$ & $\begin{array}{l}\text { Sample size ranged } \\
\text { from } 24 \text { to } 1563 ; \\
\text { this review focused } \\
\text { on adult population; } \\
\text { most of the study } \\
\text { participants were } \\
\text { male, recruited from } \\
\text { shelters, hostels, } \\
\text { missions, } \\
\text { communities, and } \\
\text { healthcare settings }\end{array}$ & $\begin{array}{l}\text { Among the included studies, } 4 \% \text { to } 7 \% \text { of } \\
\text { homeless people exhibit global cognitive } \\
\text { deficits with focal deficits in verbal and visual } \\
\text { memory, attention, speed of cognitive } \\
\text { processing, and executive functions }\end{array}$ \\
\hline $\begin{array}{l}\text { (Embleton } \\
\text { et al., } \\
\text { 2013) }\end{array}$ & $\begin{array}{l}\text { Scopus, MEDLINE, } \\
\text { Embase, Social } \\
\text { Sciences Abstract, } \\
\text { PsycINFO and } \\
\text { additional sources; } \\
\text { inception of } \\
\text { respective databases } \\
\text { to } 2011\end{array}$ & $\begin{array}{l}\text { 50; Meta- } \\
\text { analysis }\end{array}$ & $\begin{array}{l}\text { All studies were } \\
\text { selected from } \\
\text { resource- } \\
\text { constrained } \\
\text { settings ( } \mathrm{n}=22 \\
\text { countries); most } \\
\text { studies ( } \mathrm{n}=17) \\
\text { were from } \\
\text { Africa, followed } \\
\text { by South and } \\
\text { Central America } \\
(\mathrm{n}=14), \text { Asia (n } \\
=12), \text { Europe (n } \\
=5), \text { and Middle } \\
\text { East }(\mathrm{n}=2)\end{array}$ & $\begin{array}{l}\text { The total sample } \\
\text { size was } 16,987 \\
\text { (ranged from } 21 \text { to } \\
2807 \text { ); this review } \\
\text { focused on children, } \\
75 \% \text { were boys; } \\
\text { most studies did not } \\
\text { report recruitment } \\
\text { strategies, } 25 \% \text { and } \\
21 \% \text { of the samples } \\
\text { were recruited from } \\
\text { off-street and on- } \\
\text { street locations, } \\
\text { respectively }\end{array}$ & $\begin{array}{l}\text { The pooled prevalence of lifetime drug use was } \\
60 \% \text { (95\% CI: } 53-68) \text {, which was highest in } \\
\text { the Middle East ( } 83 \%, 95 \% \text { CI: } 79-87) \text { and } \\
\text { lowest in Africa ( } 47 \%, 95 \% \text { CI: } 31-64) \text { as } \\
\text { found in the location subgroups. The } \\
\text { prevalence rates for specific substance use was } \\
\text { reported for the inhalants (47\%, 95\% CI: } 36- \\
58 \text { ), tobacco (44\%, 95\% CI: } 34-55) \text {, alcohol } \\
\text { (41\%, 95\% CI: } 31-50) \text {, marijuana (31\%, 95\% } \\
\text { CI: } 18-44) \text {, and cocaine (7\%, 95\% CI: 5-9) } \\
\text { were reported }\end{array}$ \\
\hline
\end{tabular}




\begin{tabular}{|c|c|c|c|c|c|}
\hline $\begin{array}{l}\text { (Hodgson } \\
\text { et al., } \\
\text { 2013) }\end{array}$ & $\begin{array}{l}\text { Web of Science, } \\
\text { PubMed, PsycINFO, } \\
\text { and additional } \\
\text { sources; 2000-2012 }\end{array}$ & $\begin{array}{l}46 ; \\
\text { Systematic } \\
\text { review }\end{array}$ & $\begin{array}{l}\text { Studies were } \\
\text { conducted in the } \\
\text { United States ( } \mathrm{n} \\
=34) \text {, followed } \\
\text { by Canada ( } \mathrm{n}= \\
8), \text { Australia (n } \\
=6), \text { United } \\
\text { Kingdom }(\mathrm{n}= \\
\text { 2), Switzerland } \\
(\mathrm{n}=1), \text { and } \\
\text { Sweden }(\mathrm{n}=1)\end{array}$ & $\begin{array}{l}\text { Sample size ranged } \\
\text { from } 60 \text { to } 326,073 ; \\
\text { youth aged } 15-24 \\
\text { were focused in this } \\
\text { review and } \\
\text { respective studies; } \\
\text { recruited from } \\
\text { multiple sources } \\
\text { including shelters, } \\
\text { hostels, streets, and } \\
\text { support } \\
\text { organizations }\end{array}$ & $\begin{array}{l}\text { The prevalence of mental disorders ranged } \\
\text { from } 48.4 \% \text { to } 98 \% \text { across samples. Homeless } \\
\text { people had high rates of depression (17.6- } \\
28.1 \%) \text {, bipolar disorder }(26.9 \%) \text {, anxiety } \\
(32 \%) \text {, mood disorders }(12.2-41.3 \%) \text {, attention } \\
\text { deficit hyperactivity disorder }(4.4 \%) \text {, conduct } \\
\text { disorder (36-76.7\%), substance or alcohol use } \\
\text { disorder (11-43.7\%), self-harm (69\%), suicidal } \\
\text { ideation ( } 22-36.8 \%) \text {, suicidal attempts ( } 8.8- \\
46 \%) \text {. The prevalence of psychiatric } \\
\text { comorbidity in posttraumatic stress disorder } \\
\text { ranged from } 48-80.9 \% .40-67.3 \% \text { of homeless } \\
\text { individuals had co-existing substance use } \\
\text { disorder and posttraumatic stress disorder. }\end{array}$ \\
\hline $\begin{array}{l}\text { (Ennis et } \\
\text { al., 2015) }\end{array}$ & $\begin{array}{l}\text { MEDLINE/PubMed } \\
\text { (1966-2013), } \\
\text { PsycINFO (1887- } \\
\text { 2011), CINAHL } \\
(1983-2011), \text { and } \\
\text { additional sources }\end{array}$ & $\begin{array}{l}11 ; \\
\text { Systematic } \\
\text { review }\end{array}$ & $\begin{array}{l}\text { Most studies } \\
\text { were from the } \\
\text { US }(n=9), \\
\text { remaining from } \\
\text { Canada }(n=2)\end{array}$ & $\begin{array}{l}\text { Sample size ranged } \\
\text { from } 12 \text { to } 116 \text {; } \\
\text { most were men; the } \\
\text { mean age was } 40.75 \\
\text { years; participants } \\
\text { were recruited from } \\
\text { homeless shelters, } \\
\text { residential services, } \\
\text { and healthcare } \\
\text { centers }\end{array}$ & $\begin{array}{l}\text { 18-55.4\% homeless participants had general } \\
\text { cognitive deficits, } 33-69 \% \text { had verbal memory } \\
\text { impairments, and } 33-78 \% \text { had visual memory } \\
\text { impairments }\end{array}$ \\
\hline $\begin{array}{l}\text { (Bassuk et } \\
\text { al., 2015) }\end{array}$ & $\begin{array}{l}\text { Web of Science, } \\
\text { PubMed, PsycINFO. } \\
\text { and additional } \\
\text { sources; 1990-2013 }\end{array}$ & $\begin{array}{l}11 \text {; Meta- } \\
\text { analysis }\end{array}$ & $\begin{array}{l}\text { Studies } \\
\text { conducted in the } \\
\text { United States } \\
\text { were included in } \\
\text { this review }\end{array}$ & $\begin{array}{l}\text { Sample size ranged } \\
\text { from } 53 \text { to } 264 \\
\text { across homeless } \\
\text { samples; this review } \\
\text { recruited primary } \\
\text { studies focusing on } \\
\text { children and } \\
\text { adolescents from } \\
\text { multiple sources } \\
\text { shelters and housing } \\
\text { programs }\end{array}$ & $\begin{array}{l}\text { The overall prevalence of mental disorders was } \\
10-26 \% \text { among pre-schoolers and } 14-40 \% \\
\text { among school-age children who are homeless. } \\
\text { In the meta-analysis, the difference in } \\
\text { prevalence was not significantly different } \\
\text { between homeless and housed preschool } \\
\text { children (Odds ratio [OR] }=1.49 ; 95 \% \\
\text { confidence interval [CI]: } 0.97-2.28 \text { ). School- } \\
\text { age homeless children were significantly more } \\
\text { likely to have a mental health problem (OR = } \\
1.78 ; 95 \% \text { CI: } 1.19-2.66 \text { ) compared to housed } \\
\text { children. Among individual studies, the }\end{array}$ \\
\hline
\end{tabular}




\begin{tabular}{|c|c|c|c|c|c|}
\hline & & & & & $\begin{array}{l}\text { prevalence rates of depression }(13.8-46.3 \%) \\
\text { anxiety }(10-28.4 \%) \text {, conduct problems }(36 \%) \text {, } \\
\text { disruptive behavioral disorder }(19.7 \%) \text {, } \\
\text { hyperactivity problems }(34 \%) \text {, and mood } \\
\text { disorder }(5.1 \%) \text { were reported }\end{array}$ \\
\hline $\begin{array}{l}\text { (Depp et } \\
\text { al., 2015) }\end{array}$ & $\begin{array}{l}\text { PubMed, PsycINFO, } \\
\text { and additional } \\
\text { sources; 1980-2013 }\end{array}$ & $\begin{array}{l}\text { 24; Meta- } \\
\text { analysis }\end{array}$ & $\begin{array}{l}\text { Studies were } \\
\text { conducted in the } \\
\text { US }(\mathrm{n}=10) \text {, US } \\
(\mathrm{n}=5) \text {; two } \\
\text { studies each } \\
\text { from Australia, } \\
\text { Brazil, and } \\
\text { Canada; one } \\
\text { study each from } \\
\text { Spain and } \\
\text { Germany }\end{array}$ & $\begin{array}{l}\text { Total sample size } \\
\text { was } 2969 \text { (ranged } \\
\text { from } 29 \text { to } 328 \text { with } \\
\text { a mean of } 129 \text { ); } \\
\text { mean age of the } \\
\text { participants was } \\
46.1 \text { (SD 6.2) years; } \\
\text { the proportion of } \\
\text { men was } 83.2 \% \\
\text { across samples; this } \\
\text { review focused on } \\
\text { adults; the } \\
\text { participants were } \\
\text { recruited from } \\
\text { multiple locations } \\
\text { including shelters, } \\
\text { meal provision sites, } \\
\text { streets, and social } \\
\text { service settings }\end{array}$ & 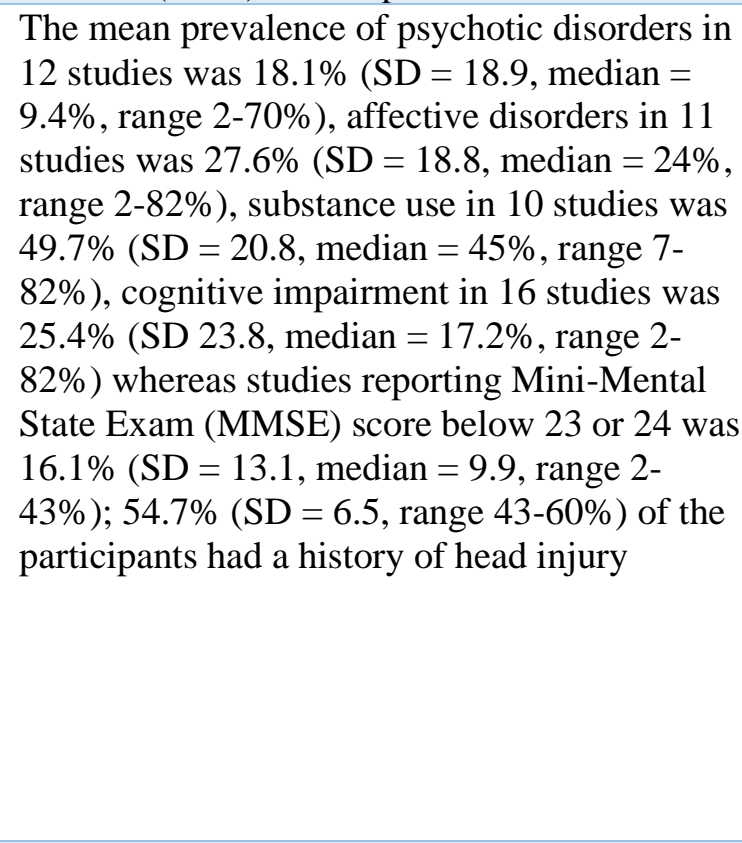 \\
\hline $\begin{array}{l}\text { (Schreiter } \\
\text { et al., } \\
\text { 2017) }\end{array}$ & $\begin{array}{l}\text { MEDLINE (1946- } \\
\text { 2016), Embase and } \\
\text { Embase Classic } \\
\text { (1947-2016), } \\
\text { PsycINFO, } \\
\text { PSYNDEX, } \\
\text { SocINDEX (1910- } \\
\text { 2016), and } \\
\text { additional sources }\end{array}$ & $\begin{array}{l}24 \text { articles } \\
\text { (11 studies); } \\
\text { Meta- } \\
\text { analysis }\end{array}$ & $\begin{array}{l}\text { Studies } \\
\text { conducted in } \\
\text { Germany were } \\
\text { included in this } \\
\text { review }\end{array}$ & $\begin{array}{l}\text { The total sample } \\
\text { size was } 1220 \\
\text { (ranged from } 17 \text { to } \\
\text { 265); mean age } \\
\text { ranged from } 29 \text { to } \\
48.1 \text { years; only two } \\
\text { samples had all } \\
\text { women participants, } \\
\text { whereas six samples } \\
\text { had no women } \\
\text { participants; } \\
\text { participants were }\end{array}$ & $\begin{array}{l}\text { In the random-effects model, the pooled } \\
\text { prevalence of mental illness was } 77.5 \% \text { (95\% } \\
\text { CI: } 72.4-82.3) \text {. The prevalence of axis I } \\
\text { disorders was } 77.4 \% \text { ( } 95 \% \text { CI: } 71.3-82.9) \text {. } \\
\text { Moreover, the prevalence rates for substance- } \\
\text { related disorders }(60.9 \%, 95 \% \text { CI: } 53.1-68.5) \text {, } \\
\text { alcohol dependency (55.4\%, } 95 \% \text { CI: } 49.2- \\
61.5) \text {, drug dependence }(13.9 \%, 95 \% \text { CI: } 7.2- \\
22.2) \text {, anxiety disorders }(17.6 \%, 95 \% \text { CI: } 12.9 \text { - } \\
22.8) \text {, affective disorder }(15.2 \%, 95 \% \text { CI: } 9.8- \\
21.5) \text {, major depression }(11.6 \%, 95 \% \text { CI: } 4.4- \\
21.3) \text {, psychotic illness }(8.3 \%, 95 \% \text { CI: } 5.4-\end{array}$ \\
\hline
\end{tabular}




\begin{tabular}{|c|c|c|c|c|c|}
\hline & & & & $\begin{array}{l}\text { recruited from } \\
\text { homeless shelters, } \\
\text { common locations, } \\
\text { and other sources } \\
\text { from different } \\
\text { German cities }\end{array}$ & $\begin{array}{l}11.8) \text {, cognitive impairment }(11.7 \%, 95 \% \mathrm{CI} \text { : } \\
6-18.9) \text {, personality disorders }(29.1 \%, 95 \% \mathrm{CI} \text { : } \\
5.6-59.5)\end{array}$ \\
\hline $\begin{array}{l}\text { (Smartt et } \\
\text { al., 2019) }\end{array}$ & $\begin{array}{l}\text { Embase, MEDLINE, } \\
\text { PsycINFO, } \\
\text { PsycArticles, Global } \\
\text { Health and Ovid } \\
\text { journals, and } \\
\text { additional sources; } \\
\text { studies published till } \\
2018\end{array}$ & $\begin{array}{l}49 \\
\text { Systematic } \\
\text { scoping } \\
\text { review }\end{array}$ & $\begin{array}{l}\text { Seven studies } \\
\text { reported the } \\
\text { prevalence of } \\
\text { mental illness, } \\
\text { among which } \\
\text { two were } \\
\text { conducted in } \\
\text { Brazil, and one } \\
\text { study each from } \\
\text { Colombia, } \\
\text { Ethiopia, } \\
\text { Nigeria, Peru, } \\
\text { Turkey }\end{array}$ & $\begin{array}{l}\text { Samples comprised } \\
\text { of diverse } \\
\text { demographic } \\
\text { characteristics; } \\
\text { recruited from } \\
\text { multiple sources } \\
\text { including shelters, } \\
\text { social programs, and } \\
\text { outdoor locations }\end{array}$ & $\begin{array}{l}\text { The prevalence of severe mental disorders } \\
\text { ranged from } 8 \% \text { to } 47.4 \%\end{array}$ \\
\hline $\begin{array}{l}\text { (Ayano, } \\
\text { Tsegay, et } \\
\text { al., 2019) }\end{array}$ & $\begin{array}{l}\text { Embase, PubMed, } \\
\text { Scopus, and } \\
\text { additional sources; } \\
\text { timeframe was not } \\
\text { specified }\end{array}$ & $\begin{array}{l}20 ; \text { Meta- } \\
\text { analysis }\end{array}$ & $\begin{array}{l}\text { Studies were } \\
\text { from the US (n } \\
=12 \text { ), Canada (n } \\
=4 \text { ), and one } \\
\text { study each from } \\
\text { Australia, Japan, } \\
\text { Ethiopia, and } \\
\text { Ghana }\end{array}$ & $\begin{array}{l}\text { Sample size ranged } \\
\text { from } 60 \text { to } 10,111 ; \\
\text { demographics and } \\
\text { recruitment strategy } \\
\text { of individual studies } \\
\text { were not specified }\end{array}$ & $\begin{array}{l}\text { The pooled prevalence of current suicidal } \\
\text { ideation among homeless individuals was } \\
17.83 \% \text { ( } 95 \% \text { CI: } 10.73-28.14 \text { ) whereas the } \\
\text { prevalence of lifetime suicidal ideation was } \\
41.6 \% \text { ( } 95 \% \text { CI: } 28.55-55.95) \text {; the pooled } \\
\text { prevalence rates of current and lifetime } \\
\text { suicidal attempt were } 9.16 \% \text { (96\% CI: } 4.1 \text { - } \\
19.2 \text { ) and } 28.8 \% \text { ( } 95 \% \text { CI: } 21.66-37.18) \text {; no } \\
\text { significant difference in the prevalence of } \\
\text { lifetime suicidal attempts was found based on } \\
\text { the quality of the studies }\end{array}$ \\
\hline $\begin{array}{l}\text { (Ayano, } \\
\text { Tesfaw, et } \\
\text { al., 2019) }\end{array}$ & $\begin{array}{l}\text { Embase, PubMed, } \\
\text { Scopus, and } \\
\text { additional sources; } \\
\text { timeframe was not } \\
\text { specified }\end{array}$ & $\begin{array}{l}31 \text {; Meta- } \\
\text { analysis }\end{array}$ & $\begin{array}{l}\text { Studies were } \\
\text { conducted in the } \\
\text { US }(n=12) \\
\text { Canada }(n=4) \text {, } \\
\text { Germany }(n= \\
\text { 3), two studies }\end{array}$ & $\begin{array}{l}\text { Sample size ranged } \\
\text { from } 33 \text { to } 29,143 ; \\
\text { demographics and } \\
\text { recruitment strategy } \\
\text { of individual studies } \\
\text { were not specified }\end{array}$ & $\begin{array}{l}\text { The pooled prevalence of psychotic disorder in } \\
\text { homeless people was } 21.21 \% \text { ( } 95 \% \text { CI: } 13.73- \\
31.29 \text { ); pooled prevalence rates of } \\
\text { schizophrenia, schizophreniform disorder, } \\
\text { schizoaffective disorder, and psychotic } \\
\text { disorder not otherwise specified (NOS) were }\end{array}$ \\
\hline
\end{tabular}


each in Ethiopia,

France, and

Scotland; one

study each in

China, Spain

UK, Ireland,

Japan, Serbia,

and Australia

\begin{tabular}{|c|c|c|c|c|c|}
\hline & & & & & the quality of the studies \\
\hline $\begin{array}{l}\text { (Duke \& } \\
\text { Searby, } \\
\text { 2019) }\end{array}$ & $\begin{array}{l}\text { CINAHL, } \\
\text { PsycINFO, PubMed, } \\
\text { Scopus, and } \\
\text { additional sources; } \\
\text { 1996-2017 }\end{array}$ & $\begin{array}{l}15 ; \\
\text { Systematic } \\
\text { review }\end{array}$ & $\begin{array}{l}\text { Studies were } \\
\text { from the US ( } \mathrm{n} \\
=10 \text { ), Canada ( } \mathrm{n} \\
=2 \text { ), Spain ( } \mathrm{n} \\
=1 \text { ) }\end{array}$ & $\begin{array}{l}\text { Sample size ranged } \\
\text { from } 8 \text { to } 821 \text {; this } \\
\text { review focused on } \\
\text { homeless women; } \\
11 \text { studies reported } \\
\text { women with } \\
\text { children; samples } \\
\text { were recruited from } \\
\text { multiple locations } \\
\text { including shelters, } \\
\text { food programs, and } \\
\text { outdoor locations }\end{array}$ & $\begin{array}{l}\text { The prevalence rates for schizophrenia }(2.5- \\
17.1 \%) \text {, drug or alcohol dependence }(7.8- \\
60 \%) \text {, posttraumatic stress disorder }(29.1- \\
41.4 \%) \text {, major depressive episode or disorder } \\
(15.8-57.9 \%) \text { were reported; } 18.3 \% \text { sample had } \\
\text { dual diagnoses in a study included in this } \\
\text { review }\end{array}$ \\
\hline
\end{tabular}

10.29\% (95\% CI: 6.44-16.02), 2.48\% (95\% CI: $0.16-28.11), 3.53 \%$ (95\% CI: $1.33-9.05)$, and 9\% (95\% CI: 6.92-11.62), respectively. In sensitivity analyses, developing countries had higher prevalence than developed countries (29.16\% compared to $18.8 \%$ for psychotic disorders, and $22.15 \%$ compared to $8.83 \%$ for schizophrenia), screening tools revealed higher prevalence than diagnostic instruments (32.19\% compared to $17.42 \%$ ); both for psychotic disorder and schizophrenia, the prevalence rates were higher for studies published on/after 2010 compared to studies published before 2010; no significant difference in prevalence rates was found for the quality of the studies $1.4 \%)$, major depressive episode or disorder dual diagnoses in a study included in this outdoor locations 


\section{Characteristics of the study populations}

The study populations in this review were homeless individuals with diverse characteristics, as identified in different study samples. The sample size among studies ranged from eight to 326,073 (Duke \& Searby, 2019; Hodgson et al., 2013). Three reviews focused on adult participants who were homeless (Burra et al., 2009; Depp et al., 2015; Spence et al., 2004). Three reviews homeless children and adolescents (Bassuk et al., 2015; Embleton et al., 2013; Parks et al., 2007). One review included primary studies with youth participants aged from 15 to 24 years (Hodgson et al., 2013). Moreover, one review focused on studies with women participants only (Duke \& Searby, 2019). In many samples, racial and ethnic minorities were over-represented within the homeless groups compared to the general population (Spence et al., 2004). The study participants were recruited from multiple sources, including homeless shelters, social services, temporary residential facilities, downtown locations, suburban areas, healthcare facilities, community locations, and streets (Bassuk et al., 2015; Burra et al., 2009; Duke \& Searby, 2019; Embleton et al., 2013; Ennis et al., 2015; Fazel et al., 2008; Folsom \& Jeste, 2002; Parks et al., 2007; Schreiter et al., 2017; Smartt et al., 2019; Spence et al., 2004).

\section{Prevalence of mental disorders among homeless people}

The prevalence of mental disorders varied across homeless samples. For example, Hodgson and colleagues reported the overall prevalence of mental disorders ranged from $48.4 \%$ to $98 \%$ among primary studies included in that review (Hodgson et al., 2013). In a meta-analysis by Schreiter and colleagues, the pooled prevalence of mental disorders was found as 77.5\% (95\% CI: 72.482.3) (Schreiter et al., 2017). The prevalence rates varied across geographic regions of corresponding primary studies among the reviews. For example, Fazel and colleagues found that primary studies conducted in the US had lower pooled prevalence of mental disorders (9\%, 95\% CI: 6-12) compared to samples from Mainland Europe (12\%, 95\% CI: 7-16), UK (19\%, 95\% CI: 9-29) and Australia (16\%, 95\% CI: 10-22) (Fazel et al., 2008). Such variations were also noted between studies from high-income countries and LMICs, studies published in the last decade and earlier years, and for different screening measures used across primary studies (Ayano, Tesfaw, et al., 2019). Moreover, reviews reported different prevalence rates of specific mental disorders among homeless populations, which are presented in the subsequent sections. 


\section{Depressive disorders and anxiety disorders}

Six reviews reported the prevalence of depressive disorders among homeless people, which ranged from 11.4\% to 57.9\% (Bassuk et al., 2015; Duke \& Searby, 2019; Fazel et al., 2008; Hodgson et al., 2013; Schreiter et al., 2017; Spence et al., 2004). For example, Bassuk and colleagues found $13.8 \%$ to $46.3 \%$ of the participants in the primary studies had depressive disorders (Bassuk et al., 2015). Another review reported $17.6 \%$ to $28.1 \%$ of the study samples were suffering from depression (Hodgson et al., 2013). Moreover, three reviews reported the prevalence of anxiety disorders, which ranged from 10\% to 32\% across samples (Bassuk et al., 2015; Hodgson et al., 2013; Schreiter et al., 2017). For example, a meta-analysis by Schreiter and colleagues found the pooled prevalence of anxiety disorders was $17.6 \%$ (95\% CI: 12.9-22.8) among homeless participants (Schreiter et al., 2017).

\section{Schizophrenia spectrum and other psychotic disorders}

Seven reviews reported the prevalence of schizophrenia spectrum and other psychotic disorders, which ranged from 1\% to 45\% (Ayano, Tesfaw, et al., 2019; Depp et al., 2015; Duke \& Searby, 2019; Fazel et al., 2008; Folsom \& Jeste, 2002; Schreiter et al., 2017; Spence et al., 2004). For example, a systematic review found that young participants had higher rates of schizophrenia (13\% to $21 \%$ ) than older participants ( $8 \%$ to $14 \%$ ), women participants had higher rates up to $35 \%$ compared to men ( 8 to $12 \%$ ), and chronically homeless individuals had higher prevalence (18\% to $27 \%$ ) compared to newly homeless individuals (2\% to $14 \%$ ) (Folsom \& Jeste, 2002). Another meta-analytic review reported the pooled prevalence of psychotic disorder in homeless people was $21.21 \%$ (95\% CI: 13.73-31.29), whereas the pooled prevalence rates of schizophrenia, schizophreniform disorder, schizoaffective disorder, and psychotic disorder not otherwise specified (NOS) were 10.29\% (95\% CI: 6.44-16.02), 2.48\% (95\% CI: 0.16-28.11), 3.53\% (95\% CI: 1.33-9.05), and 9\% (95\% CI: 6.92-11.62), respectively (Ayano, Tesfaw, et al., 2019).

\section{Substance-related and addictive disorders}

In this umbrella review, seven reviews were identified that reported the prevalence of substancerelated and addictive disorders, which ranged from $4.5 \%$ to $60.9 \%$ across homeless samples (Depp et al., 2015; Duke \& Searby, 2019; Embleton et al., 2013; Fazel et al., 2008; Hodgson et 
al., 2013; Schreiter et al., 2017; Spence et al., 2004). For example, a meta-analytic review found the pooled prevalence of alcohol dependence was 37.9\% (95\% CI: 27.8-48) (Fazel et al., 2008). Another review found that $11 \%$ to $43.7 \%$ had alcohol or other substance use disorder (Hodgson et al., 2013).

\section{Neurocognitive disorders}

Six reviews reported the prevalence of neurocognitive disorders, which ranged from $4 \%$ to $80 \%$ (Burra et al., 2009; Depp et al., 2015; Ennis et al., 2015; Parks et al., 2007; Schreiter et al., 2017; Spence et al., 2004). The most commonly reported problems were cognitive impairments among homeless people. For example, Ennis and colleagues found $18 \%$ to $55.4 \%$ homeless participants had general cognitive deficits (Ennis et al., 2015). A meta-analytic review found 25.4\% of the homeless adults had cognitive impairment (Depp et al., 2015). Another review by Parks and colleagues found $11 \%$ to $35 \%$ of homeless children had impaired cognitive functions and associated disabilities (Parks et al., 2007).

\section{Bipolar disorders and mood disorders}

Four reviews reported the prevalence of bipolar and mood disorders among homeless people, which ranged from 5.1\% to 41.3\% (Bassuk et al., 2015; Depp et al., 2015; Hodgson et al., 2013; Schreiter et al., 2017). A review by Hodgson and colleagues found $26.9 \%$ of homeless participants had bipolar disorders (Hodgson et al., 2013). Moreover, 12.2\% to $41.3 \%$ of homeless samples had mood disorders in this review. Furthermore, a review by Depp and colleagues reported the prevalence of affective or mood disorders was $27.6 \%$ ( $\mathrm{SD}=18.8$, median $=24 \%$ ) in 10 studies (Depp et al., 2015).

\section{Suicidal behavior disorder}

Two reviews identified prevalence rates for suicidal ideation, attempt, and self-injury among homeless people (Ayano, Tsegay, et al., 2019; Hodgson et al., 2013). Hodgson and colleagues reported the prevalence rates of self-harm (69\%), suicidal ideation (22-36.8\%), and suicidal attempts (8.8-46\%) (Hodgson et al., 2013). Another review by Ayano and colleagues reported the pooled prevalence of current suicidal ideation was $17.83 \%$ (95\% CI: 10.73-28.14), whereas the prevalence of lifetime suicidal ideation was $41.6 \%$ (95\% CI: 28.55-55.95) (Ayano, Tsegay, et 
al., 2019). Moreover, the pooled prevalence rates of current and lifetime suicidal attempts were 9.16\% (96\% CI: 4.1-19.2) and 28.8\% (95\% CI: 21.66-37.18), respectively.

\section{Other mental disorders among homeless people}

Several other mental disorders were reported across reviews. Two reviews reported the

prevalence of attention-deficit/hyperactivity disorder and conduct disorder ranging from $4.4 \%$ to $34 \%$ and $36 \%$ to $76.7 \%$, respectively (Bassuk et al., 2015; Hodgson et al., 2013). Moreover, two reviews reported that $23.1 \%$ to $29.1 \%$ of homeless people had personality disorders (Fazel et al., 2008; Schreiter et al., 2017). Furthermore, Bassuk and colleagues found $19.7 \%$ of children and adolescents had disruptive behavioral disorders. A review by Smartt and colleagues found seven studies from LMICs, which reported $8 \%$ to $47.4 \%$ of the homeless samples had severe mental disorders (Smartt et al., 2019). Another review by Duke and Searby reported that $29.1 \%$ to $41.4 \%$ of homeless women had posttraumatic stress disorder, whereas Hodgson and colleagues found the prevalence of psychiatric comorbidity in posttraumatic stress disorder ranged from $48 \%$ to $80.9 \%$. Moreover, $40 \%$ to $67.3 \%$ of homeless individuals had co-existing substance use disorder and posttraumatic stress disorder. Such co-existence of multiple mental disorders and other clinical conditions was reported in several reviews (Depp et al., 2015; Duke \& Searby, 2019; Hodgson et al., 2013; Smartt et al., 2019; Spence et al., 2004).

\section{Discussion}

\section{Overview of synthesized findings of this umbrella review}

To our knowledge, this is the first umbrella review reporting the overall prevalence of different mental disorders among people who are homeless. The synthesized findings from existing evidence-based reviews inform a high burden of depressive disorders, anxiety disorders, schizophrenia spectrum and psychotic disorders, bipolar and mood disorders, substance use disorders, suicidal behavior and self-injury, posttraumatic stress disorders, neurocognitive disorders, and other psychiatric conditions. In addition to the adults, high prevalence rates were found among children and adolescents who are homeless. Fewer studies reported high prevalence rates of mental disorders among homeless women (Duke \& Searby, 2019). Most reviews had a higher number of primary studies from high-income countries, and the proportion 
of racial and ethnic minorities was higher in many reviews (Bassuk et al., 2015; Fazel et al., 2008; Schreiter et al., 2017; Spence et al., 2004). The prevalence rates were different across study samples, which necessitates an in-depth evaluation of the potential reasons contributing to such heterogeneity to better understand the findings of this review.

Psychiatric research on the homeless population is often constrained by several factors, which may have affected the existing evidence base in this domain. The working definition of homelessness may differ across contexts, which may affect the estimation of homeless individuals and evaluate any health problems among this mobile and vulnerable population (Fazel, Geddes, \& Kushel, 2014; Williams, 2017). For example, a study in the US evaluated how changed definitions impacted estimations of homeless populations and found that a change in the definitions excluded nearly half of the chronically homeless individuals (Byrne \& Culhane, 2015). Moreover, definitions and measurements of homelessness may also result in inaccurate estimations of mental health conditions. A study found that objectively defined homelessness was associated with higher rates of alcohol use and substance use disorders compared to subjectively reported homelessness (Eyrich-Garg, O'Leary, \& Cottler, 2008). These differences are critical as the psychosocial epidemiology can be uniquely different among the included or excluded individuals based on such changes. It is essential to consider these issues while using the findings of this study as well as conducting future research in this domain.

Another issue is the changing definitions of mental disorders and instruments measuring the same, which may have resulted in different prevalence estimations across studies (Ayano, Tesfaw, et al., 2019). Also, the low number of reviews and the median number of databases suggest a need to synthesize robust evidence from more data sources focusing on diverse mental health outcomes among homeless people.

The geographic and contextual focus of many studies provided evidence for those areas, whereas many nations with a high number of homeless populations may remain under-examined among the existing reviews, which include countries in South America, Sub-Saharan Africa, and South Asia. This can be a result of a gap in evidence synthesis or a critical lack of primary studies conducted in those regions.

Furthermore, the co-existence of multiple mental disorders as well as physical comorbidity among the homeless with mental disorders highlight the severity of disability across samples 
(Depp et al., 2015; Duke \& Searby, 2019; Hodgson et al., 2013; Spence et al., 2004). This may inform inadequate evidence if the primary studies measured only one or a few mental health conditions rather than a thorough evaluation of multiple health problems among the study samples.

Finally, limited evidence is found on how the mental health status changed among people before and after experiencing homelessness or how different the prevalence rates are between the homeless samples and the general population within the same geographic and sociocultural contexts. These issues should be considered to contextualize the findings of the current review and to inform future knowledge synthesis.

\section{Implications for future research, policy development, and practice}

The findings of this review provide valuable insights to conduct future research, adopt appropriate policies, and improve psychosocial care through better practice. First, longitudinal studies using standardized research instruments should be used to evaluate the mental health conditions and associated factors among homeless people, which may further improve the quantity and quality of knowledge in this area and inform evidence-based practice.

Second, psychopathological processes among socio-economically marginalized populations who are vulnerable to homelessness should be examined. For example, people who experienced forced migration and associated psychosocial trauma may experience higher burden of mental disorders while living in temporary shelters or unstable social settings (Hossain \& Purohit, 2018). It is essential to identify various psychosocial factors associated with mental health resilience and outcomes during homelessness, which may help in preventing mental disorders among homeless people through early psychosocial interventions (Hughes et al., 2010; Lee, Liang, Rotheram-Borus, \& Milburn, 2011).

Third, it is necessary to examine how different mental disorders evolve over time and change their courses among homeless populations. Multiple psychiatric diagnoses among people who are already experiencing one or more neuropsychiatric conditions is a major global mental health concern (Hossain, Khan, et al., 2020; Hossain, Purohit, et al., 2020). In addition, homeless individuals generally experience instability in terms of residential, occupational, cultural, social, and environmental aspects, which is likely to exert a compounding effect of psychosocial burden 
among those individuals. Therefore, the prognosis of mental disorders among homeless may not be similar to the general population living in comparatively stable conditions (Dawson \& Jackson, 2013; Hodgson et al., 2013; Stubbs et al., 2019). Such variability among mental health prognosis may inform the psychiatrists, psychologists, social workers, and other caregivers to provide adequate support based on the individual mental health needs.

Fourth, little is known about mental health-seeking behavior among homeless individuals. Identifying the barriers and facilitators of individuals and groups in terms of seeking mental care can be a potential research agenda for future studies, which may inform effective policy development in mental health. In this discourse, homelessness and associated mental health outcomes should be evaluated in respective contexts, especially how these constructs are defined and conceptualized in those places. It is necessary to assess and compare how such problems are different than the general population and what are the determinants of mental health in those contexts. Such contextualization of evidence may inform better decision-making to address mental health gaps across homeless populations.

Fifth, health services research should be conducted to examine how health systems and existing modalities of mental health care are equipped to address the burden of mental disorders among homeless people. This may require policy analyses and evaluations of mental health as well as social care programs to assess the effectiveness of the existing approaches, identify the gaps through rigorous research, and address the same through evidence-based multipronged pharmacological and non-pharmacological interventions (Baxter, Tweed, Katikireddi, \& Thomson, 2019; Fitzpatrick-Lewis et al., 2011).

Last but not least, improving the mental health outcomes among homeless people would necessitate addressing the underlying causes of homelessness and persistent psychosocial stressors (Embleton, Lee, Gunn, Ayuku, \& Braitstein, 2016; Nooe \& Patterson, 2010), which should be prioritized across communities and organizations. A meaningful partnership among major stakeholders can mobilize resources alleviating homelessness and improve mental health among homeless individuals. 


\section{Limitations}

This umbrella review has several limitations. One such limitation is a potential selection bias as we did not search all the databases and excluded unpublished studies and reports. Another limitation is the publication bias within the scientific literature as less significant findings are less likely to get published, thus may not contribute to the evidence base. Moreover, we did not conduct a quantitative evaluation of the patient-level data, which could have eliminated betweenstudy and within-study variations and provided uniform evidence of prevalence estimations across samples. However, this umbrella review was conducted using systematic guidelines involving more than two reviewers to ensure scientific rigor at each stage of review. Future evidence synthesis should address the existing limitations and advance the knowledge base in this domain.

\section{Conclusion}

Homelessness is a prevalent problem across societies with enormous psychosocial impacts on population health. This umbrella review systematically evaluated the current evidence on the prevalence of mental disorders among homeless people. The findings of this review inform a high epidemiological burden of mental disorders in homelessness, which requires multi-level interventions to address the same. Moreover, future research should be conducted to improve the evidence base on psychopathological correlates, processes, and outcomes associated with homelessness across populations. The current evidence on mental health problems warrants a better understanding of the underlying socioeconomic challenges that impact overall health and wellbeing. Nonetheless, the definitions of homelessness and mental health conditions continue to evolve across places and over time, which is a continued challenge for synthesizing knowledge and translating the same in practice. It is essential to acknowledge and address the methodological and contextual issues that may inform a better understanding of mental health among homeless populations. Lastly, evidence-based insights should be translated to future policies, programs, and services envisaging effective prevention, diagnosis, and treatment of mental disorders among people who are homeless. 
Declaration of interests: We declare no conflicts of interest.

Acknowledgment: None.

Role of the funding source: No funding was received at any stage of conducting this umbrella review or preparing this manuscript.

\section{References}

Aldridge, R. W., Menezes, D., Lewer, D., Cornes, M., Evans, H., Blackburn, R. M., ... Hayward, A. (2019). Causes of death among homeless people: a population-based cross-sectional study of linked hospitalisation and mortality data in England. Wellcome Open Research, 4, 49. https://doi.org/10.12688/wellcomeopenres.15151.1

Aldridge, R. W., Story, A., Hwang, S. W., Nordentoft, M., Luchenski, S. A., Hartwell, G., ... Hayward, A. C. (2018). Morbidity and mortality in homeless individuals, prisoners, sex workers, and individuals with substance use disorders in high-income countries: a systematic review and meta-analysis. The Lancet, 391(10117), 241-250. https://doi.org/10.1016/S0140-6736(17)31869-X

Altena, A. M., Brilleslijper-Kater, S. N., \& Wolf, J. L. M. (2010, June). Effective Interventions for Homeless Youth. A Systematic Review. American Journal of Preventive Medicine. https://doi.org/10.1016/j.amepre.2010.02.017

American Psychiatric Association. (2013). Diagnostic and Statistical Manual of Mental Disorders (DSM-5). Retrieved December 5, 2019, from https://www.psychiatry.org/psychiatrists/practice/dsm

Aromataris, E., Fernandez, R., Godfrey, C. M., Holly, C., Khalil, H., \& Tungpunkom, P. (2015). Summarizing systematic reviews: methodological development, conduct and reporting of an umbrella review approach review of reviews, systematic review, umbrella review, umbrella review methodology. International Journal of Evidence-Based Healthcare. https://doi.org/10.1097/XEB.0000000000000055

Ayano, G., Tesfaw, G., \& Shumet, S. (2019). The prevalence of schizophrenia and other 
psychotic disorders among homeless people: A systematic review and meta-analysis. $B M C$ Psychiatry, 19. Retrieved from http://proxy.library.tamu.edu/login?url=http://search.ebscohost.com/login.aspx?direct=true $\& \mathrm{db}=$ psyh \&AN=2019-73871-001\&site=ehost-live

Ayano, G., Tsegay, L., Abraha, M., \& Yohannes, K. (2019). Suicidal Ideation and Attempt among Homeless People: a Systematic Review and Meta-Analysis. Psychiatric Quarterly, 90(4), 829-842. Retrieved from http://10.0.3.239/s11126-019-09667-8

Bassuk, E. L., Richard, M. K., \& Tsertsvadze, A. (2015). The Prevalence of Mental Illness in Homeless Children: A Systematic Review and Meta-Analysis. Journal of the American Academy of Child \& Adolescent Psychiatry, 54(2), 86-96. Retrieved from http://10.0.3.248/j.jaac.2014.11.008

Bastian, H., Glasziou, P., \& Chalmers, I. (2010). Seventy-five trials and eleven systematic reviews a day: How will we ever keep up? PLoS Medicine, 7(9). https://doi.org/10.1371/journal.pmed.1000326

Baxter, A. J., Tweed, E. J., Katikireddi, S. V., \& Thomson, H. (2019). Effects of Housing First approaches on health and well-being of adults who are homeless or at risk of homelessness: systematic review and meta-analysis of randomised controlled trials. Journal of Epidemiology And Community Health, 73(5), 379-387. https://doi.org/10.1136/jech-2018210981

Burra, T. A., Stergiopoulos, V., \& Rourke, S. B. (2009). A systematic review of cognitive deficits in homeless adults: Implications for service delivery. Canadian Journal of Psychiatry, 54(2), 123-133. https://doi.org/10.1177/070674370905400210

Byrne, T., \& Culhane, D. P. (2015). Testing alternative definitions of chronic homelessness. Psychiatric Services, 66(9), 996-999. https://doi.org/10.1176/appi.ps.201400240

Daund, M., Sonavane, S., Shrivastava, A., Desousa, A., \& Kumawat, S. (2018, February 1). Mental Hospitals in India: Reforms for the future. Indian Journal of Psychiatry. Medknow Publications. https://doi.org/10.4103/psychiatry.IndianJPsychiatry_434_17

Dawson, A., \& Jackson, D. (2013). The primary health care service experiences and needs of 
homeless youth: A narrative synthesis of current evidence. Contemporary Nurse: A Journal for the Australian Nursing Profession, 44(1), 62-75. Retrieved from http://10.0.20.52/conu.2013.44.1.62

Depp, C. A., Vella, L., Orff, H. J., \& Twamley, E. W. (2015). A quantitative review of cognitive functioning in homeless adults. Journal of Nervous and Mental Disease, 203(2), 126-131. https://doi.org/10.1097/NMD.0000000000000248

Dickey, B. (2000). Review of programs for persons who are homeless and mentally ill. Harvard Review of Psychiatry, 8(5), 242-250. Retrieved from http://www.ncbi.nlm.nih.gov/pubmed/11118233

Duke, A., \& Searby, A. (2019). Mental Ill Health in Homeless Women: A Review. Issues in Mental Health Nursing, 40(7), 605-612. Retrieved from http://10.0.4.56/01612840.2019.1565875

Embleton, L., Lee, H., Gunn, J., Ayuku, D., \& Braitstein, P. (2016). Causes of Child and Youth Homelessness in Developed and Developing Countries: A Systematic Review and Metaanalysis. JAMA Pediatrics, 170(5), 435-444. https://doi.org/10.1001/jamapediatrics.2016.0156

Embleton, L., Mwangi, A., Vreeman, R., Ayuku, D., \& Braitstein, P. (2013). The epidemiology of substance use among street children in resource-constrained settings: a systematic review and meta-analysis. Addiction, 108(10), 1722-1733. Retrieved from http://10.0.4.87/add.12252

Ennis, N., Roy, S., \& Topolovec-Vranic, J. (2015). Memory impairment among people who are homeless: A systematic review. Memory, 23(5), 695-713. Retrieved from http://10.0.4.56/09658211.2014.921714

Eyrich-Garg, K. M., O’Leary, C. C., \& Cottler, L. B. (2008). Subjective Versus Objective Definitions of Homelessness: Are There Differences in Risk Factors Among HeavyDrinking Women? Gender Issues, 25(3), 173-192. https://doi.org/10.1007/s12147-0089057-5

Fazel, S., Geddes, J. R., \& Kushel, M. (2014, October 25). The health of homeless people in 
high-income countries: Descriptive epidemiology, health consequences, and clinical and policy recommendations. The Lancet. Lancet Publishing Group. https://doi.org/10.1016/S0140-6736(14)61132-6

Fazel, S., Khosla, V., Doll, H., \& Geddes, J. (2008). The Prevalence of Mental Disorders among the Homeless in Western Countries: Systematic Review and Meta-Regression Analysis. PLoS Medicine, 5(12), e225. Retrieved from http://10.0.5.91/journal.pmed.0050225

Fitzpatrick-Lewis, D., Ganann, R., Krishnaratne, S., Ciliska, D., Kouyoumdjian, F., \& Hwang, S. W. (2011). Effectiveness of interventions to improve the health and housing status of homeless people: A rapid systematic review. BMC Public Health. https://doi.org/10.1186/1471-2458-11-638

Folsom, D., \& Jeste, D. V. (2002). Schizophrenia in homeless persons: a systematic review of the literature. Acta Psychiatrica Scandinavica, 105(6), 404-413. Retrieved from http://10.0.4.10/j.1600-0447.2002.02209.x

Fullana, M. A., Tortella-Feliu, M., Fernández De La Cruz, L., Chamorro, J., Pérez-Vigil, A., Ioannidis, J. P. A., ... Radua, J. (2019). Risk and protective factors for anxiety and obsessive-compulsive disorders: An umbrella review of systematic reviews and metaanalyses. Psychological Medicine. Cambridge University Press. https://doi.org/10.1017/S0033291719001247

Gaetz, S., Dej, E., Richter, T., \& Redman, M. (2016). The State of Homelessness in Canada 2016. Retrieved from www.homelesshub.ca

Henry, M., Mahathey, A., Morrill, T., Robinson, A., Shivji, A., \& Watt, R. (2018). The 2018 Annual Homeless Assessment Report (AHAR) to Congress, Part 1: Point-in-Time Estimates of Homelessness.

Hodgson, K. J., Shelton, K. H., van den Bree, M. B. M., \& Los, F. J. (2013). Psychopathology in Young People Experiencing Homelessness: A Systematic Review. American Journal of Public Health, 103(6), e24-e37. Retrieved from http://10.0.8.57/AJPH.2013.301318

Hossain, M. M., Khan, N., Sultana, A., Ma, P., McKyer, E. L. J., Ahmed, H. U., \& Purohit, N. (2020). Prevalence of comorbid psychiatric disorders among people with autism spectrum 
disorder: An umbrella review of systematic reviews and meta-analyses. Psychiatry

Research, 112922. https://doi.org/10.1016/j.psychres.2020.112922

Hossain, M. M., \& Purohit, N. (2018, February 10). Protecting Rohingya: lives, minds, and the future. The Lancet. Lancet Publishing Group. https://doi.org/10.1016/S01406736(18)30209-5

Hossain, M. M., Purohit, N., Sultana, A., Ma, P., J McKyer, E. L., \& Uddin Ahmed, H. (2020). Prevalence of mental disorders in South Asia: an umbrella review of systematic reviews and meta-analyses. Asian Journal of Psychiatry, 102041. https://doi.org/10.1016/j.ajp.2020.102041

Hughes, J. R., Clark, S. E., Wood, W., Cakmak, S., Cox, A., Macinnis, M., ... Broom, B. (2010). Youth Homelessness: The Relationships among Mental Health, Hope, and Service Satisfaction. Journal of the Canadian Academy of Child and Adolescent Psychiatry = Journal de l'Academie Canadienne de Psychiatrie de l'enfant et de l'adolescent, 19(4), 274-283. Retrieved from http://www.ncbi.nlm.nih.gov/pubmed/21037918

Ioannidis, J. P. A. (2009). Integration of evidence from multiple meta-analyses: A primer on umbrella reviews, treatment networks and multiple treatments meta-analyses. CMAJ, 181(8), 488-493. https://doi.org/10.1503/cmaj.081086

Jones, M. M. (2016). Does Race Matter in Addressing Homelessness? A Review of the Literature. World Medical and Health Policy, 8(2), 139-156. https://doi.org/10.1002/wmh3.189

Lee, S. J., Liang, L. J., Rotheram-Borus, M. J., \& Milburn, N. G. (2011). Resiliency and survival skills among newly homeless adolescents: Implications for future interventions. Vulnerable Children and Youth Studies, 6(4), 301-308. https://doi.org/10.1080/17450128.2011.626468

Liberati, A., Altman, D. G., Tetzlaff, J., Mulrow, C., Gotzsche, P. C., Ioannidis, J. P., ... Moher, D. (2009). The PRISMA statement for reporting systematic reviews and meta-analyses of studies that evaluate healthcare interventions: explanation and elaboration. Bmj, 339, b2700. https://doi.org/10.1136/bmj.b2700

Munn, Z., Tufanaru, C., \& Aromataris, E. (2014). JBI's systematic reviews: data extraction and 
synthesis. The American Journal of Nursing, 114(7), 49-54.

https://doi.org/10.1097/01.NAJ.0000451683.66447.89

Nooe, R. M., \& Patterson, D. A. (2010). The Ecology of Homelessness. Journal of Human Behavior in the Social Environment, 20(2), 105-152. https://doi.org/10.1080/10911350903269757

Omerov, P., Craftman, Å. G., Mattsson, E., \& Klarare, A. (2020). Homeless persons' experiences of health- and social care: A systematic integrative review. Health \& Social Care in the Community, 28(1), 1-11. https://doi.org/10.1111/hsc.12857

Parks, R. W., Stevens, R. J., \& Spence, S. A. (2007). A systematic review of cognition in homeless children and adolescents. Journal of the Royal Society of Medicine, 100(1), 4650. https://doi.org/10.1258/jrsm.100.1.46

Patten, S. B. (2017). Homelessness and Mental Health. Canadian Journal of Psychiatry, 62(7), 440-441. https://doi.org/10.1177/0706743717711423

Rosenheck, R., \& Seibyl, C. L. (1998). Homelessness: Health Service Use and Related Costs. Medical Care. Lippincott Williams \& Wilkins. https://doi.org/10.2307/3766891

Schreiter, S., Bermpohl, F., Krausz, M., Leucht, S., Rössler, W., Schouler-Ocak, M., \& Gutwinski, S. (2017). The Prevalence of Mental Illness in Homeless People in Germany: A Systematic Review and Meta-analysis. Deutsches Aerzteblatt International, 114(40), 665672. https://doi.org/10.3238/arztebl.2017.0665

Shelter England. (2018). 320,000 people in Britain are now homeless, as numbers keep rising Shelter England. Retrieved January 12, 2020, from https://england.shelter.org.uk/media/press_releases/articles/320,000_people_in_britain_are_ now_homeless,_as_numbers_keep_rising

Smartt, C., Prince, M., Frissa, S., Eaton, J., Fekadu, A., \& Hanlon, C. (2019). Homelessness and severe mental illness in low- and middle-income countries: scoping review. Bjpsych Open, 5(4), e57-e57. https://doi.org/10.1192/bjo.2019.32

Speak, S. (2019). The State of Homelessness in Developing Countries. 
Spence, S., Stevens, R., \& Parks, R. (2004, August). Cognitive dysfunction in homeless adults: A systematic review. Journal of the Royal Society of Medicine.

https://doi.org/10.1258/jrsm.97.8.375

Stubbs, J. L., Thornton, A. E., Sevick, J. M., Silverberg, N. D., Barr, A. M., Honer, W. G., \& Panenka, W. J. (2019). Traumatic brain injury in homeless and marginally housed individuals: a systematic review and meta-analysis. The Lancet. Public Health. https://doi.org/10.1016/S2468-2667(19)30188-4

United Nations. (2015). Transforming our world: the 2030 Agenda for Sustainable Development.

US Health and Human Services. (2020). Definitions of Homelessness for Federal Program Serving Children, Youth, and Families.

Williams, J. C. (2017). The Politics of Homelessness in the United States (Vol. 1). Oxford University Press. https://doi.org/10.1093/oxfordhb/9780199935307.013.153

World Health Organization (WHO). (2016). International Classification of Diseases (ICD-10) Version:2016. Retrieved December 5, 2019, from https://icd.who.int/browse10/2016/en

Yim, L. C. L., Leung, H. C. M., Chan, W. C., Lam, M. H. B., \& Lim, V. W. M. (2015). Prevalence of mental illness among homeless people in Hong Kong. PLoS ONE, 10(10). https://doi.org/10.1371/journal.pone.0140940

Yohanna, D. (2013). Deinstitutionalization of people with mental illness: Causes and consequences. Virtual Mentor. American Medical Association. https://doi.org/10.1001/virtualmentor.2013.15.10.mhst1-1310 
Supplemental file: Critical appraisal of the systematic reviews and meta-analyses

\begin{tabular}{|c|c|c|c|c|c|c|c|c|c|c|c|}
\hline Source & $\begin{array}{l}\text { Is the } \\
\text { review } \\
\text { question } \\
\text { clearly } \\
\text { and } \\
\text { explicitly } \\
\text { stated? }\end{array}$ & $\begin{array}{l}\text { Were the } \\
\text { inclusion } \\
\text { criteria } \\
\text { appropri } \\
\text { ate for } \\
\text { the } \\
\text { review } \\
\text { question? }\end{array}$ & $\begin{array}{l}\text { Was the } \\
\text { search } \\
\text { strategy } \\
\text { appropri } \\
\text { ate? }\end{array}$ & $\begin{array}{l}\text { Were the } \\
\text { sources } \\
\text { and } \\
\text { resources } \\
\text { used to } \\
\text { search for } \\
\text { studies } \\
\text { adequate? }\end{array}$ & $\begin{array}{l}\text { Were the } \\
\text { criteria for } \\
\text { appraising } \\
\text { studies } \\
\text { appropriate } \\
\text { ? }\end{array}$ & $\begin{array}{l}\text { Was critical } \\
\text { appraisal } \\
\text { conducted } \\
\text { by two or } \\
\text { more } \\
\text { reviewers } \\
\text { independen } \\
\text { tly? }\end{array}$ & $\begin{array}{l}\text { Were the } \\
\text { methods used } \\
\text { to combine } \\
\text { studies } \\
\text { appropriate? }\end{array}$ & $\begin{array}{l}\text { Was the } \\
\text { likelihood } \\
\text { of } \\
\text { publicatio } \\
\text { n bias } \\
\text { assessed? }\end{array}$ & $\begin{array}{l}\text { Were } \\
\text { recommend } \\
\text { ations for } \\
\text { policy } \\
\text { and/or } \\
\text { practice } \\
\text { supported } \\
\text { by the } \\
\text { reported } \\
\text { data? }\end{array}$ & $\begin{array}{l}\text { Were the } \\
\text { specific } \\
\text { directives } \\
\text { for new } \\
\text { research } \\
\text { appropria } \\
\text { te? }\end{array}$ & $\begin{array}{l}\text { Overall } \\
\text { score and } \\
\text { quality } \\
\text { rating }\end{array}$ \\
\hline $\begin{array}{l}\text { (Folsom \& } \\
\text { Jeste, 2002) }\end{array}$ & Yes & Yes & Yes & Yes & No & No & Yes & No & Yes & Yes & $\begin{array}{l}7 \\
\text { (medium) }\end{array}$ \\
\hline $\begin{array}{l}\text { (Spence et al., } \\
\text { 2004) }\end{array}$ & Yes & Yes & Yes & Yes & No & No & Yes & No & Yes & Yes & $\begin{array}{l}7 \\
\text { (medium) }\end{array}$ \\
\hline $\begin{array}{l}\text { (Parks et al., } \\
\text { 2007) }\end{array}$ & Yes & Yes & Yes & Yes & No & Yes & No & No & Yes & Yes & $\begin{array}{l}7 \\
\text { (medium) }\end{array}$ \\
\hline $\begin{array}{l}\text { (Fazel et al., } \\
\text { 2008) }\end{array}$ & Yes & Yes & Yes & Yes & Yes & CD & Yes & No & Yes & Yes & 8 (High) \\
\hline $\begin{array}{l}\text { (Burra et al., } \\
\text { 2009) }\end{array}$ & Yes & Yes & Yes & Yes & Yes & Yes & Yes & No & Yes & Yes & 9 (High) \\
\hline $\begin{array}{l}\text { (Embleton et } \\
\text { al., 2013) }\end{array}$ & Yes & Yes & Yes & Yes & $\mathrm{CD}$ & No & Yes & No & Yes & Yes & $\begin{array}{l}7 \\
\text { (medium) }\end{array}$ \\
\hline $\begin{array}{l}\text { (Hodgson et al., } \\
\text { 2013) }\end{array}$ & Yes & Yes & Yes & Yes & No & Yes & $\mathrm{CD}$ & No & Yes & Yes & $\begin{array}{l}7 \\
\text { (medium) }\end{array}$ \\
\hline $\begin{array}{l}\text { (Ennis et al., } \\
\text { 2015) }\end{array}$ & Yes & Yes & Yes & Yes & Yes & $\mathrm{CD}$ & Yes & No & $\mathrm{CD}$ & Yes & $\begin{array}{l}7 \\
\text { (medium) }\end{array}$ \\
\hline
\end{tabular}




\begin{tabular}{|c|c|c|c|c|c|c|c|c|c|c|c|}
\hline $\begin{array}{l}\text { (Bassuk et al., } \\
\text { 2015) }\end{array}$ & Yes & Yes & Yes & Yes & Yes & Yes & Yes & No & Yes & Yes & 9 (High) \\
\hline $\begin{array}{l}\text { (Depp et al., } \\
\text { 2015) }\end{array}$ & Yes & Yes & Yes & Yes & $\mathrm{CD}$ & Yes & Yes & No & Yes & Yes & 8 (High) \\
\hline $\begin{array}{l}\text { (Schreiter et } \\
\text { al., 2017) }\end{array}$ & Yes & Yes & Yes & Yes & No & No & Yes & No & Yes & Yes & $\begin{array}{l}7 \\
\text { (medium) }\end{array}$ \\
\hline $\begin{array}{l}\text { (Smartt et al., } \\
\text { 2019) }\end{array}$ & Yes & Yes & Yes & Yes & Yes & Yes & Yes & No & Yes & Yes & 9 (High) \\
\hline $\begin{array}{l}\text { (Ayano, } \\
\text { Tsegay, et al., } \\
\text { 2019) }\end{array}$ & Yes & Yes & Yes & Yes & Yes & Yes & Yes & Yes & Yes & Yes & 10 (High) \\
\hline $\begin{array}{l}\text { (Ayano, } \\
\text { Tesfaw, et al., } \\
\text { 2019) }\end{array}$ & Yes & Yes & Yes & Yes & Yes & Yes & Yes & Yes & Yes & Yes & 10 (High) \\
\hline $\begin{array}{l}\text { (Duke \& } \\
\text { Searby, 2019) }\end{array}$ & Yes & Yes & Yes & Yes & Yes & No & Yes & No & Yes & Yes & 8 (High) \\
\hline
\end{tabular}

